\title{
Role of the Convection Scheme in Modeling Initiation and Intensification of Tropical Depressions over the North Atlantic
}

\author{
J.-P. DUVEL \\ Laboratoire de Météorologie Dynamique, CNRS, École Normale Supérieure, Paris, France \\ S. J. CAMARGO \\ Lamont-Doherty Earth Observatory, Columbia University, Palisades, New York
}

A. H. SOBEL

Lamont-Doherty Earth Observatory, Palisades, and Department of Applied Physics and Applied Mathematics, Columbia University, New York, New York

(Manuscript received 6 June 2016, in final form 22 December 2016)

\begin{abstract}
The authors analyze how modifications of the convective scheme modify the initiation of tropical depression vortices (TDVs) and their intensification into stronger warm-cored tropical cyclone-like vortices (TCs) in global climate model (GCM) simulations. The model's original convection scheme has entrainment and cloud-base mass flux closures based on moisture convergence. Two modifications are considered: one in which entrainment is dependent on relative humidity and another in which the closure is based on the convective available potential energy (CAPE).

Compared to reanalysis, TDVs are more numerous and intense in all three simulations, probably as a result of excessive parameterized deep convection at the expense of convection detraining at midlevel. The relative humidity-dependent entrainment rate increases both TDV initiation and intensification relative to the control. This is because this entrainment rate is reduced in the moist center of the TDVs, giving more intense convective precipitation, and also because it generates a moister environment that may favor the development of early stage TDVs. The CAPE closure inhibits the parameterized convection in strong TDVs, thus limiting their development despite a slight increase in the resolved convection. However, the maximum intensity reached by TC-like TDVs is similar in the three simulations, showing the statistical character of these tendencies.

The simulated TCs develop from TDVs with different dynamical origins than those observed. For instance, too many TDVs and TCs initiate near or over southern West Africa in the GCM, collocated with the maximum in easterly wave activity, whose characteristics are also dependent on the convection scheme considered.
\end{abstract}

\section{Introduction}

Variations in the large-scale environment may have important impacts on tropical cyclone (TC) activity, whether those variations occur on intraseasonal [Madden-Julian oscillation (MJO)] or interannual [El Niño-Southern Oscillation (ENSO)] time scales, or in response to longer-term global climate change. The sensitivity of TCs to the large-scale environment can now be studied using global climate models (GCMs; see e.g., Walsh et al. 2016; Camargo and Wing 2016). Cyclogenesis is a complex process, however, and it is not trivial to

Corresponding author e-mail: J.-P. Duvel, jpduvel@lmd.ens.fr determine the causes of variations in TC activity, either in nature or in a GCM. Considering early vortices' initiation and intensification processes separately can potentially lead to a better assessment of the ability of GCMs to correctly reproduce the sensitivity of TCs to the largescale environment.

A tropical cyclone may indeed form locally by convective aggregation processes (not necessarily well represented in a GCM), or it can be triggered dynamically by preexisting disturbances or vortices. In the "vortex view" of TC genesis (Davis et al. 2008), the vortices are seen as possible TC seeds that can be initiated by tropical waves or by other mechanisms, related, for example, to orography (Mozer and Zehnder 1996) 
well before their intensification to TC strength. If a high percentage of TCs in a basin are initiated from these vortices, then the physical source of these vortices becomes an important TC assessment criterion. By using either GCM outputs or meteorological analysis combined with TC observation databases, it is possible to study the environmental conditions during the formation of vortices-referred to here as tropical depression vortices (TDVs) - which can serve as TC seeds. For example, previous studies (Liebmann et al. 1994; Duvel 2015) have shown that the MJO's modulation of TC frequency over the Indian Ocean is mainly due to its modulation of the number of TDVs and only marginally to its modulation of intensification processes. We are interested here in applying the same approach to understand the sources of variability in TC characteristics simulated by different GCM formulations. Over the North Atlantic, African easterly waves (AEWs) are known to be sources of cyclogenetic TDVs near the West African coast (e.g., Landsea 1993; Dunkerton et al. 2009) and can be an important factor in the ability of GCMs to simulate TC activity (Daloz et al. 2012). These waves have long been studied (i.e., Carlson 1969; Burpee 1972, 1975; Reed et al. 1977), but GCMs still have difficulty in simulating AEWs, and there are still large uncertainties regarding possible modifications of AEWs due to global climate change (Martin and Thorncroft 2015). It is thus likely that part of the misrepresentation of TCs in a GCM over the North Atlantic can be related to the TDVs associated with AEWs.

With horizontal resolutions in the range of $0.1^{\circ}-1^{\circ}$, a GCM is able to simulate the initiation and intensification of TDVs. Some TDVs may become very intense for part of their path and have characteristics similar to observed tropical cyclones, even if the cyclone mesoscale structure is not well represented. It is possible to track the TDVs in a GCM and also to select only TDVs with a tropical cyclone-like vertical structure, as is done by the Camargo and Zebiak (2002, hereinafter CZO2) algorithm, which detects and tracks warm-core vortices. Previous studies have analyzed the influence of the convection scheme on the TC characteristics in GCMs with various spatial resolutions (e.g., Vitart et al. 2001; Zhao et al. 2012; Murakami et al. 2012b; Stan 2012; Kim et al. 2012). In particular, Murakami et al. (2012a) reported significant differences in TC characteristics in a $20-\mathrm{km}$-mesh model with two different versions of the convection scheme (one based on an Arakawa-Schubert scheme and one based on the Tiedtke scheme). The greater TC intensity in the Tiedtke-based scheme was mostly attributed to its stronger inhibition of the convection, which increased the gridscale resolved convection (larger upward motion and large-scale condensation) and the associated moisture supply at low levels. A previous study by Vitart et al. (2001) in a coarser model (T42) also showed that the inhibition of the convection enhanced the TC frequency, but this was mostly attributed to the effect of this inhibition on the increase of the background CAPE. As noted in Vitart et al. (2001), it is possible that larger CAPE is necessary to produce TCs when the resolution is lower, to compensate for the inhibition of vertical motion by the coarse resolution. This large CAPE can increase the number of TCs, but the most important driver for the TC intensity appears to be the horizontal resolution. Kim et al. (2012) showed that in a low-resolution $\left(2^{\circ} \times 2.5^{\circ}\right)$ GCM, the TC frequency was reduced with a larger entrainment, while another factor-the rain reevaporationwas found to increase the TC frequency. This ambiguous influence of the entrainment on the convective inhibition and TC number is perhaps consistent with the results of Zhao et al. (2012), showing that the inhibition of the convection favors TC genesis up to certain point but reduces TC genesis when the entrainment is too strong. This was attributed to the fact that the resolved convection at first enhances the TC activity but then can also counteract the formation of coherent vortices by favoring the spatial noisiness of the convection.

Other factors can also play a role in the TC frequency and intensity. For example, Stan (2012) showed that an explicit representation (the so-called superparameterization) of cloud processes in a low-resolution T42 GCM increases the TC activity compared to a conventional parameterization, by increasing the moistening of the lower troposphere (850-700 hPa). Reed and Jablownowski (2011) showed that the growth of an idealized vortex (early stage TDV) depends on both the spatial resolution and the manner in which the CAPE (defining the closure of the convection scheme) is calculated. Using the same approach, He and Posselt (2015) showed that among 24 different parameters, the convective entrainment rate has the largest role in TDV intensification with larger intensification for the smaller entrainment rate (i.e., an inverse effect compared to TC intensification due to the convective inhibition effect of the entrainment).

Here we use the Laboratoire de Météorologie Dynamique zoom model (LMDZ) to study the sensitivity of TDV characteristics to different entrainment and closure formulations of the convection scheme. This study uses the zoom capability of the LMDZ GCM with a resolution of about $0.75^{\circ}$ over a large region of the North Atlantic and West Africa. We use the Tiedtke convection scheme either with entrainment formulation and overall closure based on moisture convergence or with an entrainment based on the relative humidity of the environment, as well as a closure based on CAPE. Each configuration is run for $10 \mathrm{yr}$ between 2000 and 


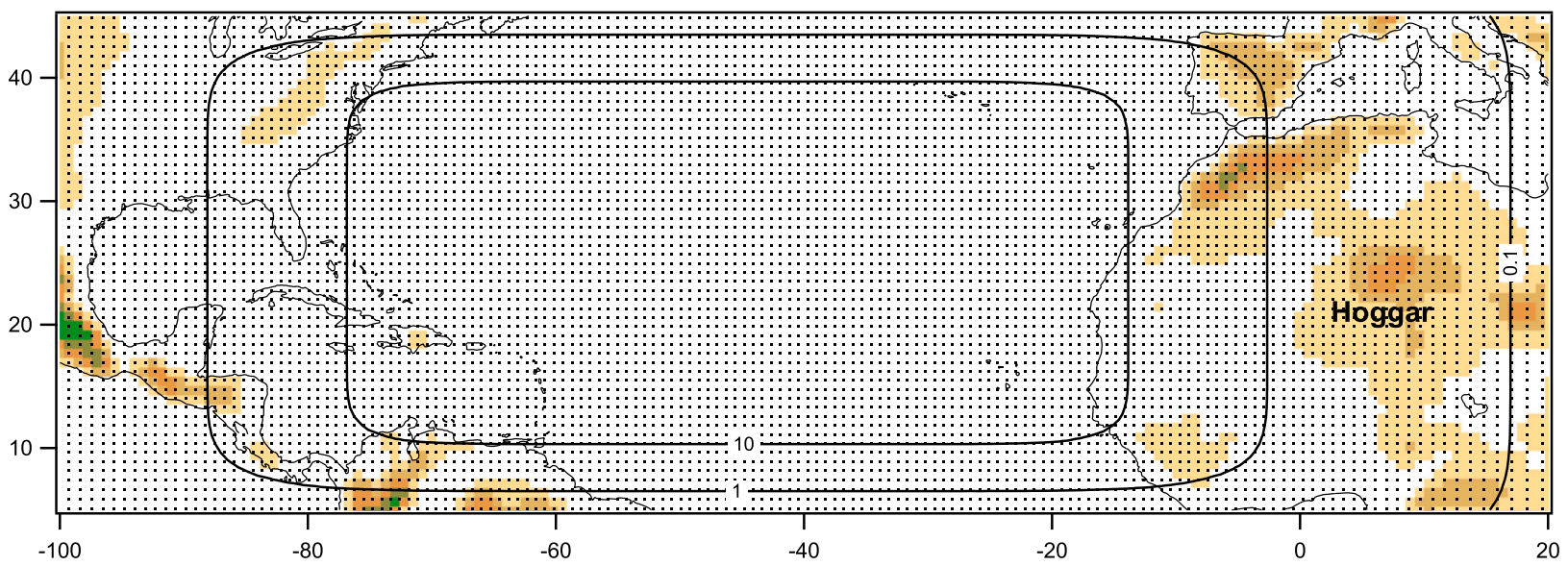

FIG. 1. Model grid points in the zoomed region (dots) and relaxation time $\tau_{u}$ in days for the wind (contours) for $\tau_{u}$ smaller or equal to 10 days $\left(\tau_{u}\right.$ continues to increase in the zoomed region, but the model may be considered as already free to run for $\tau_{u}$ greater than 10 days).

2009 with prescribed observed SST. The aim is to analyze the impact of the convection scheme on the initiation stage of the TDVs and on their probability of surviving and intensifying over the African continent and over the ocean. The assessment of the different GCM configurations is done by first comparing TDV characteristics (such as initiation, duration, strength) to those extracted from the ECMWF interim reanalyses [ERA-Interim (ERA-I); Dee et al. 2011]. The approach introduced in Duvel (2015) is used to define TDV characteristics at the same horizontal resolution of $0.75^{\circ}$ for both LMDZ and ERA-I. In parallel, the CZ02 tracking algorithm is used to assess more specifically the activity of mature tropical cyclone-like storms in the GCM in comparison with IBTrACS observations (Knapp et al. 2010).

Section 2 presents succinctly LMDZ, the zoom configuration, and the different closure and entrainment formulations of the convection scheme. The two tracking algorithms and some metrics are presented in section 3 . The distributions of TDV and TC characteristics (frequency, duration, intensity) are analyzed in section 4 . The initiation locations, the tracks, and the intensity distributions are analyzed in section 5, and the seasonal and interannual variations in section 7 . Potential physical sources of the differences between the simulations are analyzed in section 7 , and section 8 contains a summary.

\section{Model simulations}

The simulations are performed using version 4 (v.4) of LMDZ, as described in Hourdin et al. (2006). We use the Tiedtke (1989) bulk mass flux scheme for moist convection instead of the Emanuel (1991) convection scheme used in the standard LMDZ v.4 because it allows us more flexibility in modifying the closure (i.e., the cloud-base mass flux) and entrainment formulations. We use the zoom capability of LMDZ (see, e.g., Yang et al. 2016) with a resolution of about $0.75^{\circ}$ over a wide area covering the North Atlantic and part of West Africa. The domain encompasses West Africa, since this region has been shown to be important for TC simulations (Caron and Jones 2012). The model is free to run in a large central part of the zoomed region, while it is totally constrained to remain close to the ERA-I meteorological reanalyses outside of this region. There are intermediate relaxation times in the buffer zone around the zoom region (Fig. 1). The regular westward decrease of the nudging intensity over West Africa gives a smooth boundary condition (compared to a sharp lateral boundary forcing) for AEWs and TDVs generated over West Africa and that are susceptible to enter the Atlantic Ocean. This nudging also ensures comparable lateral boundary conditions for the three simulations and thus reduces differences that could be due to biased large-scale conditions around the zoomed region.

The guidance from ERA-I is applied to the wind, temperature, and humidity fields. For a field $\mathbf{x}$, the time evolution is given by

$$
\frac{\partial \mathbf{x}}{\partial t}=\left(\frac{\partial \mathbf{x}}{\partial t}\right)_{\mathrm{GCM}}+\frac{\mathbf{x}_{\mathrm{era}}-\mathbf{x}}{\tau_{x}}
$$

where the first right-hand-side term is the tendency given by the GCM and the second right-hand-side term is the relaxation toward its value in ERA-I $\left(\mathbf{x}_{\text {era }}\right)$ with a relaxation time $\tau_{x}$. Based on this principle, a relaxation increment $d x=-\alpha_{x}\left(x-x_{\text {era }}\right)$ is applied every five dynamical time steps. The relaxation factor $\alpha_{x}$ is defined as

$$
\alpha_{x}=\left(1-e^{-5 d t / \tau_{x}}\right)
$$

where $d t=45 \mathrm{~s}$ is the model time step for dynamical processes. 
For the wind, the relaxation time is set to a very large value in the heart of the zoomed region and is at a minimum value of $30 \mathrm{~min}$ outside the zoomed region (Fig. 1). The relaxation factor $\alpha_{u}$ is computed with an analytical function of the horizontal resolution with near-zero values at the center of the zoomed region and a value of 0.12 outside. The same process is applied to temperature and humidity, but with larger values of the minimum relaxation time ( $6 \mathrm{~h}$ and 3 days, respectively) in order to limit the nudging impact on thermodynamic variables over West Africa and to avoid model instabilities outside the zoomed region. For synoptic time scales of interest here, the relaxation is negligible for relaxation times greater than 10 days and is already weak for relaxation times around 2 days. We may thus consider that the wind is not significantly influenced locally by the nudging over the Atlantic and near the West African coast. As intended by our nudging approach, there is a substantial nudging of the wind near and east of the Greenwich meridian where AEW and some TDVs are initiated, giving realistic seeds for possible westward TDV and TC developments in the three LMDZ simulations. For temperature and humidity, one may consider that the nudging is negligible west of $10^{\circ} \mathrm{E}$.

The vertical redistribution of water and energy in the Tiedtke convection scheme is based on one single saturated updraft profile and one single downdraft profile extending from the free sinking level to the cloud base. The mass flux at the top of the downdraft is a constant fraction (0.3) of the convective mass flux at the cloud base. The downdraft remains saturated by evaporating precipitation. This scheme does not consider convective momentum transport, but previous studies by Reed and Jablownowski (2011) and He and Posselt (2015) showed that it has a small effect on TC intensity for models with a comparable horizontal resolution. The activation of the moist convection scheme depends on the buoyancy of the lifted parcel at the first grid level above the condensation level. In its original formulation (noted TIE here), both the closure (i.e., the value of the mass flux at the cloud base) and the entrainment of environmental air above the cloud base depend on the moisture divergence profile. Here, the scheme was modified progressively by first considering an entrainment that depends on the environmental relative humidity following the formulation described in Bechtold et al. (2008). With this new entrainment (noted ENT), the entrainment rate is larger in drier environments, inhibiting the convection, and smaller in humid environments, favoring the convection. ENT thus increases the contrast between dry and wet environments and the variability of the convective/precipitation rate compared to TIE. An additional modification (noted CAPENT) uses a closure based on CAPE, as described in Bechtold et al. (2014), but without accounting for the imbalance between boundary layer heating and deep convective overturning. With this new closure, the primary convective strength (i.e., prior to the modulation due to entrainment) does not depend on the low-level moisture convergence (as for TIE and ENT) but on the static stability of the column.

When active within a TDV or TC, the convective scheme dries and warms the atmospheric column and reinforces the vortex intensity. The surface friction under the vortex generates a low-level convergence that plays an ambiguous role in the original Tiedtke scheme (TIE) by increasing both the entrainment above the cloud base (convection weakening by mixing with the drier environment) and the mass flux at the cloud base (convection strengthening). With the new entrainment (ENT) the convection will be at the first order inhibited in dry vortices and favored in wet vortices; one may thus anticipate stronger convection and vortex intensity with ENT for strong vortices (associated with large low-level moisture convergence) with nearly saturated centers over the ocean. With the new closure (CAPENT), there is a disconnect between the low-level moisture convergence and the primary convective intensity. This disconnect is far from total, however, since in a vortex, the low-level convergence is associated with a resolved upward motion that tends to increase the temperature gradient and the CAPE. After strong convective episodes, one may expect that smaller CAPE tends to inhibit the convection for the following time steps in CAPENT. If the inhibition of the convection is too large, the center of the vortex is not dried out and may become saturated. This can lead to an unexpected resolved convection in the center of the vortex with excessive upward motion and low-level moisture convergence compared to the parameterized convection.

The three versions of the convection scheme described above-TIE, ENT, and CAPENT - are used for three AMIP-type simulations with the zoomed grid and with 39 vertical levels (only 22 levels below $20 \mathrm{~km}$ ). We performed 10-yr simulations between 2000 and 2009 forced with ERA-I fields and observed SST. It is thus possible to study the interannual variability of the TDV activity related to interannual variability of SST and of large-scale lateral conditions. As shown below, it is not trivial to identify the convective scheme giving the best TDV/TC simulation, since the score can depend on what criteria are used to evaluate the statistics of the TDVs and TCs detected by the two tracking algorithms.

\section{Tracking algorithms}

The TDV tracking is based on the approach described in Duvel (2015). For each time step (here, every 6h), a 
TDV area is defined as an ensemble of continuous grid points with geopotential height anomaly $(\Delta \phi)$ at $850 \mathrm{hPa}$ lower than a given threshold. Term $\Delta \phi$ is defined as the difference between $\phi$ and the average $\phi$ over a region of $\pm 7.5^{\circ}$ (here \pm 10 grid points). As in Duvel (2015), an empirical threshold $\Delta \phi_{0}=-80 \mathrm{~m}^{2} \mathrm{~s}^{-2}$ is set as the minimal geopotential perturbation considered. This relatively weak threshold allows for the detection of TDVs at an early stage, but stronger TC-like systems have a too large TDV area at $\Delta \phi_{0}$ with ill-defined characteristics. The TDV area is thus computed for a series of deeper thresholds (i.e., $<-80 \mathrm{~m}^{2} \mathrm{~s}^{-2}$ ), and the first threshold giving an equivalent radius of the TDV area lower than $3^{\circ}$ of latitude-longitude is retained. For developed cyclones, this threshold may be less than $-1200 \mathrm{~m}^{2} \mathrm{~s}^{-2}$.

The tracking of a given TDV is performed by considering the overlap between TDV areas in two consecutive time steps. If several TDVs are overlapping, then only the TDV with the largest overlap is considered for the continuity of the tracking. Each TDV is thus represented by a time series of characteristics of overlapping TDV areas (barycenter, maximum surface wind, maximum vorticity at $850 \mathrm{hPa}$, maximum $\Delta \phi$, minimum surface pressure, etc.).

Here, since we are mostly interested in simulating TC activity over the North Atlantic Ocean, we consider only TDVs that are initiated south of $40^{\circ} \mathrm{N}$ and that spend at least 2 days over the tropical North Atlantic waters. These TDVs are called Atlantic TDVs, or simply TDVs here. This means that TDVs that initiate over West Africa but dissipate before reaching the Atlantic are not considered. Because of the chosen nudging configuration, TDVs initiated near and east of the Greenwich meridian (see Fig. 1) are partly forced by the wind nudging toward ERA-I. However, the dissipation or the maintenance of these TDVs as they propagate westward toward the African west coast and over the Atlantic is fully determined by LMDZ.

The strength of a given TDV is characterized by accumulated cyclone energy (ACE) computed on the basis of the maximum surface wind $v_{\max }$ in the TDV area at each time step. The values in the model are not directly comparable to the observed ACE, since the maximum surface wind perturbations in the simulation and in the reanalyses are far weaker than the maximum sustained winds in real observed TCs. The formulation is, however, the same,

$$
\mathrm{ACE}=10^{-4} \sum v_{\max }^{2}
$$

where the sum is defined over every $6 \mathrm{~h}$ during the TDV lifetime and $v_{\text {max }}$ is the maximum surface $(10 \mathrm{~m})$ wind speed in the TDV area expressed in knots $\left(1 \mathrm{kt}=0.5144 \mathrm{~m} \mathrm{~s}^{-1}\right)$.
Note that this definition differs from the standard ACE in that the latter considers only steps with storm intensities larger than $35 \mathrm{kt}$ (Bell et al. 2000; Maue 2009), whereas we include all steps in which the TDV is defined by the tracking scheme. The strongest TDVs will be defined using this ACE metric on a per-storm basis. To inspect the TDV strength spatial distribution, we will also sum the ACE over all the TDVs crossing a particular region.

Tropical cyclone-like vortices are detected and tracked using the CZ02 algorithm. This tracking algorithm first identifies TC-like features with a maximum local relative vorticity $(850 \mathrm{hPa})$, minimum surface pressure, and a warm core (defined by the local temperature anomaly). To be considered as a possible TC-like storm, these features must last at least 2 days (nonconsecutive). Once these potential TCs are identified, in the second part of the algorithm, these storms are tracked using a relaxed vorticity threshold (i.e., lower than in the first part of the algorithm) by connecting the vorticity centroid every $6 \mathrm{~h}$. This algorithm has been extensively used in global (Camargo et al. 2005; Camargo and Barnston 2009; Camargo 2013; Shaevitz et al. 2014) and regional climate models (Landman et al. 2005; Camargo et al. 2007a). Here, we considered the same thresholds in all LMDZ simulations, namely, a minimum of $7.5 \times 10^{-5} \mathrm{~s}^{-1}$ (vorticity), $8.5 \mathrm{~m} \mathrm{~s}^{-1}$ (wind speed), and $1.5 \mathrm{~K}$ (temperature anomaly over a $5 \times 5$ gridpoint box) for detection, and $4.5 \times 10^{-5} \mathrm{~s}^{-1}$ vorticity for the tracking part of the algorithm.

The CZ02 scheme was initially developed to identify TC-like vortices in low-resolution models, recognizing that the simulated interannual variations of the activity in such models could be simulated well enough to be useful for prediction and some research purposes even when their intensities are well below those observed in real TCs. At the resolution considered here, many of the systems detected by the CZ02 algorithm are weaker than observed TCs and so the phrase "TC-like vortices" is still to some extent appropriate. We nonetheless denote them as "TCs" here, for brevity. The distinction we make between TCs and TDVs is that the former are defined using a wider range of criteria appropriate (qualitatively if not quantitatively) to real tropical cyclones, including a warm core, while TDVs here are defined using simpler and less restrictive criteria that allow, for example, coldcore systems and early stage vortices.

\section{TDV and TC statistics}

LMDZ tends to generate more TDVs than does ERA-I with a maximum obtained for ENT (Table 1). Considering the same constraints (systems lasting more than 2 days over ocean south of $40^{\circ} \mathrm{N}$ ), there are 139 TCs in 
TABLE 1. Statistics on the number of TDVs and TCs in observations and for the three LMDZ simulations. A match between a TC and a TDV track means that at least $50 \%$ of the TC locations are at a distance smaller than $3^{\circ}$ from a given TDV.

\begin{tabular}{lcccc}
\hline \hline & ERA-I/ & & & \\
& IBTrACS & TIE & ENT & CAPENT \\
\hline No. of TDVs $\left(N_{\text {TDV }}\right)$ & 616 & 693 & 988 & 871 \\
No. of TCs $\left(N_{\text {TC }}\right)$ & 139 & 96 & 241 & 152 \\
No. of matching & 128 & 92 & 219 & 136 \\
$\quad$ TC tracks $\left(M_{\text {TC }}\right)$ & & & & \\
TC ratio $\left(N_{\text {TC }} / N_{\text {TDV }}\right)$ & 0.23 & 0.14 & 0.24 & 0.17 \\
Match ratio $\left(M_{\text {TC }} / N_{\text {TC }}\right)$ & 0.92 & 0.96 & 0.91 & 0.89 \\
Duration TC/duration TDV & 0.77 & 0.80 & 0.74 & 0.69 \\
Pre-TC duration (days) & 2.3 & 2.6 & 3.4 & 4.4 \\
\hline
\end{tabular}

IBTrACS between 2000 and 2009. The CZ02 approach tracks fewer TCs than observed for TIE and more than observed for CAPENT and ENT. Despite the different tracking algorithms and/or the different data sources for observations, the agreement between the TC and the TDV tracking is fairly good. TC tracks with at least half of the points in common with a given TDV track represent more than $89 \%$ of the TC tracks for the simulations and $92 \%$ for observations (despite the very different tracking process for TDVs in ERA-I and TCs in IBTrACS). The
TC duration varies between $69 \%$ of the TDV duration for CAPENT and $80 \%$ for TIE compared to $77 \%$ in observations. This variability is partly related to the duration of the pre-TC phase and thus also depends on the genesis location for both TDVs and TCs.

Since the number of systems diagnosed by the tracking schemes can increase dramatically if some thresholds are relaxed (e.g., if the minimum duration is reduced from 2 days to 1 day), average TDV characteristics tend to be biased toward the more numerous weaker systems. It is therefore interesting to examine how average TDV characteristics change as the ensemble $N(20 \leq N \leq 200)$ of the strongest TDV (largest ACE) increases. The ACE is overestimated in LMDZ compared to ERA-I and is 3 times larger for ENT for $N=20$ (Fig. 2a). This is partly due to overestimated maximum wind speed (Fig. 2c) and duration (Fig. 2f). For $N<100$, the strongest ACE is obtained for ENT and TIE (Fig. 2a). The duration, the distance covered by the TDVs, and the ACE decrease, however, faster with $N$ in TIE compared to ENT and CAPENT. This is mostly related to a longer (shorter) duration for strong (weak) TDVs in TIE (Fig. 2f). The ACE also decays much more rapidly with $N$ in IBTrACS than in ERA-I or LMDZ (Fig. 2a), mostly because of the strong TC intensity contrast
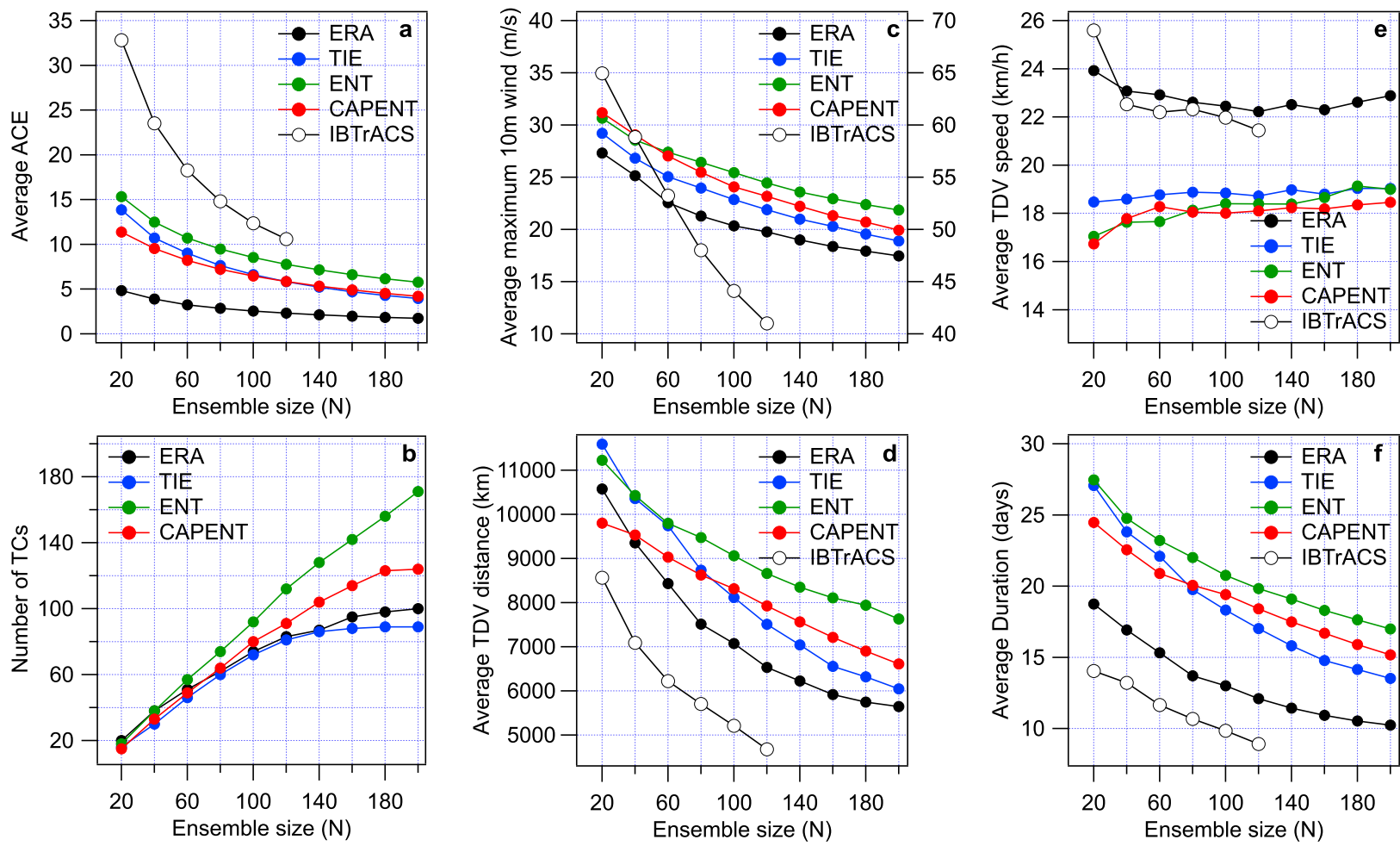

FIG. 2. TDV characteristics averaged or integrated over the $N$ systems with the largest ACE as a function of $N$ for (a) ACE, (b) number of TCs, (c) maximum wind speed, (d) distance, (e) TDV speed, and (f) duration. The results are also shown for the observed TCs [with ordinates on the right axis for (c)]. 

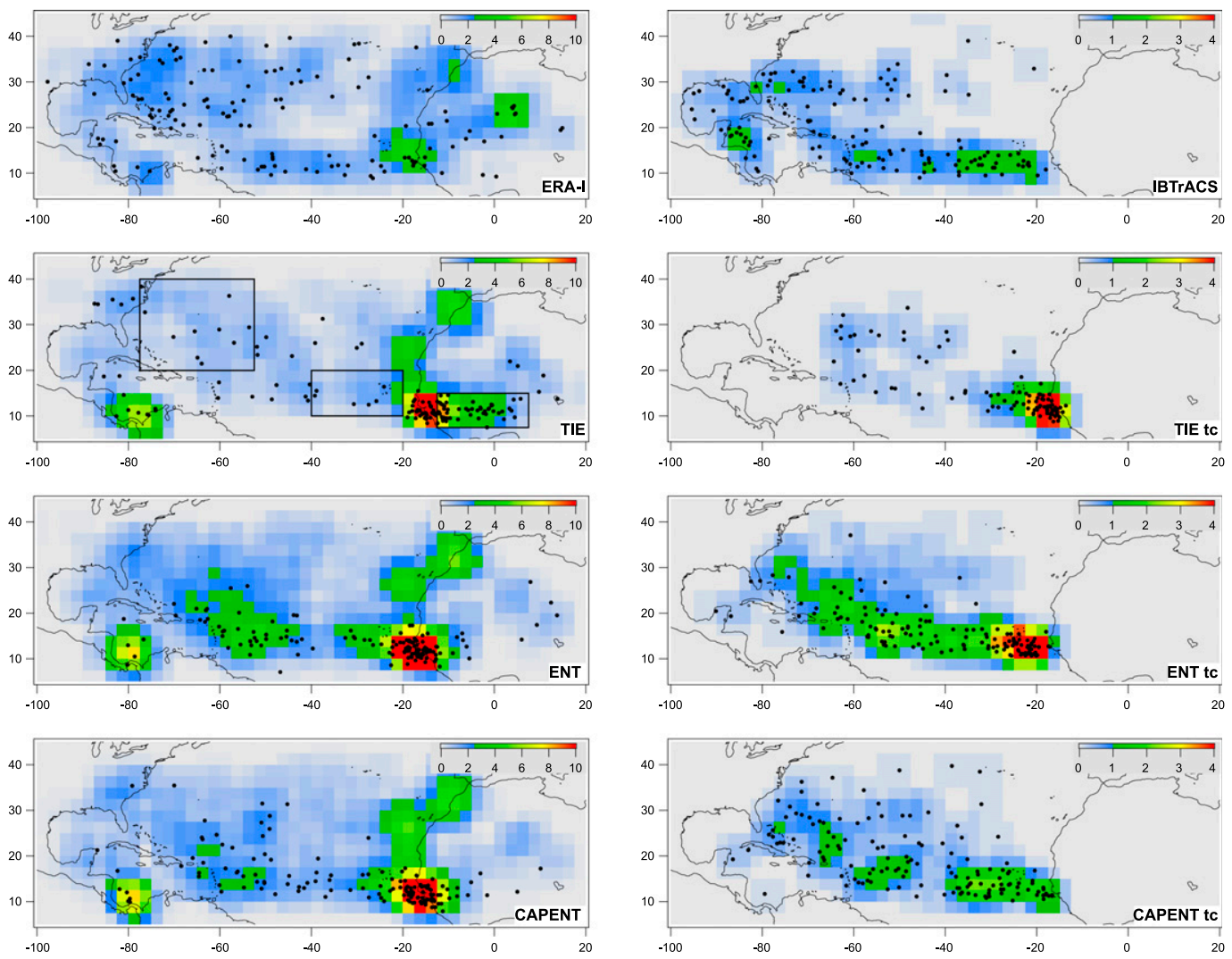

FIG. 3. Genesis density (No. $/ 2.5^{\circ}$ region) of (left) Atlantic TDVs for ERA-I and LMDZ simulations and (right) TCs for IBTrACS and LMDZ simulations for June-November 2000-09 (colors). The fields are smoothed by a $3 \times 3$ average filter for better legibility. The genesis locations for the 140 TDVs with the largest ACE are shown (black dots).

between the strongest TDVs and the others (Fig. 2c). The relatively low horizontal resolution used in LMDZ and ERA-I does not lead to such a large intensity contrast, even for those TDVs selected by the CZ02 algorithm. In fact, as shown in Fig. 2b, the first 60 TCs detected by the CZ02 algorithm in LMDZ are almost identical to the first 60 TDVs.

The average TDV speed is larger for ERA-I compared to LMDZ simulations (Fig. 2e). This speed reflects both the average large-scale steering flow and the $\beta$ effect that gives a displacement speed proportional to the square root of the vortex surface wind (e.g., Smith 1993). Figure 2e shows, interestingly, that this speed is quite comparable in ERA-I and IBTrACS despite the very different vortex wind speeds in the two datasets. This suggests that this speed is probably nudged by the assimilation procedure in ERA-I.

The larger number of TDVs for ENT and CAPENT (Table 1) means that the new entrainment enhanced the initiation of Atlantic TDVs, due to either a modification of the atmospheric background conditions (average moisture profiles, average steering flow, wind shear, etc.) or local processes within early stage vortices. The proportion of TCs is also larger for ENT and CAPENT compared to TIE (Table 1), showing that the intensification of these TDVs is also favored by the new entrainment. However, the maximum intensity reached by a TDV is very similar in the three simulations, as shown by average ACE and maximum 10-m wind along the track (Fig. 2), suggesting that the new entrainment mostly favors the intensification of early stage vortices.

Some of the strongest TDVs are initiated in the heart of West Africa, in relation to African easterly waves (Martin and Thorncroft 2015) or depressions due to the orography (Fig. 3). There are large differences in the TDV genesis density between ERA-I and LMDZ and between the different versions of the convective scheme. As expected, the primary region of TDV genesis is near the African coast around $10^{\circ} \mathrm{N}$. For LMDZ, this region concentrates the genesis of many of the 140 strongest TDVs and even the 20 strongest TDVs (Fig. 4). For TIE, the majority of the strongest TDVs form inland near the coast and further east over the Guinean region. With the new entrainment, TDV and TC initiation near the 

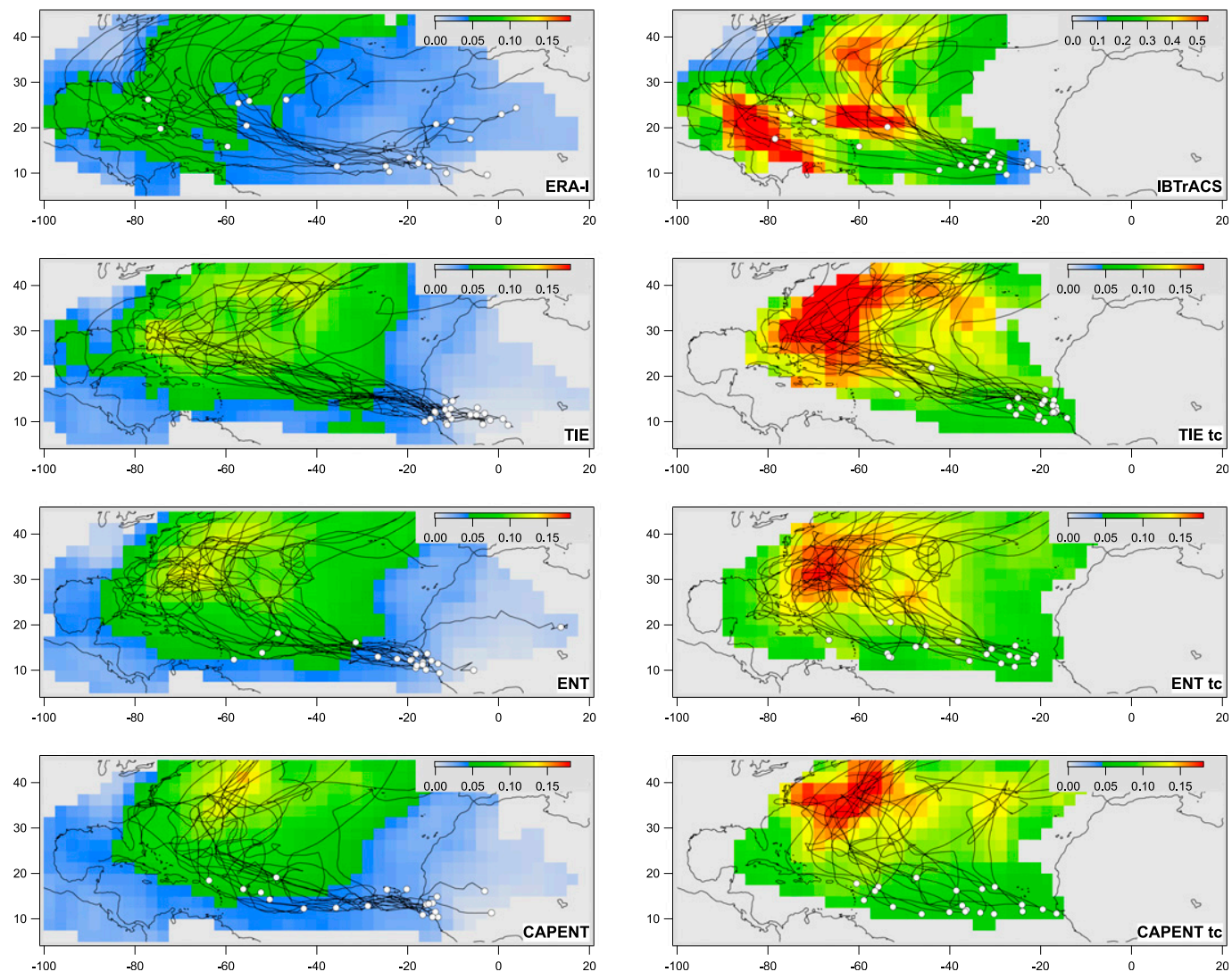

FIG. 4. Average ACE in $2.5^{\circ}$ regions of (left) Atlantic TDVs for ERA-I and LMDZ simulations and (right) TCs for IBTrACS and LMDZ simulations for June-November 2000-09 (colors). The scale is threefold for IBTrACS, and fields are smoothed by a $3 \times 3$ average filter for better legibility. Also shown are trajectories (black lines) and genesis locations (white circles) for the $20 \mathrm{TDV}$ /TCs with the largest ACE.

African coast is also exaggerated, but more TDVs and TCs are initiated over the ocean, in better agreement with observations. In LMDZ, there is a general lack of TDV and TC initiations in the western part of the domain, except for TDVs generated north of Panama.

In ERA-I, there is a secondary genesis density maximum, including some of the strongest TDVs, downwind of the Hoggar Mountains (Fig. 3), prolonged southwestward by an "initiation corridor" until the African coast. It is outside of the scope of this study to analyze in detail the genesis process for these TDVs, but it could be related to the low pressure area downwind of the Hoggar Mountains and thus related to the strength of the harmattan (a dry northeasterly wind blowing at low levels over the Sahara). In LMDZ, there are fewer TDVs forming over this region despite the relatively strong wind nudging $\left(\tau_{u}<1\right)$ near the Hoggar Mountains. This could indicate that TDVs generated in this region dissipate rapidly in LMDZ in the dry environment of the Sahel region and are thus not detected in our analysis due to the criterion requiring that the TDVs exist for 2 days over the ocean. In ERA-I, seven of the 20 strongest TDVs are initiated over continental regions of West Africa, including 5 in the initiation corridor between the Hoggar Mountains and the coast (Fig. 4). For ENT and CAPENT, most of the 20 strongest TDVs are initiated over the ocean, but for TIE, the 20 strongest TDVs all form over the continental Guinean regions around $10^{\circ} \mathrm{N}$. Because of the substantial wind nudging over the eastern Guinean region, it is likely that part of these TDVs are initiated by the wind nudging but are more persistent in TIE (even compared to ERA-I) while they move westward across the African coast.

The TDV ACE (Fig. 4) is maximum over the western Atlantic Ocean and larger for LMDZ (around 0.12) than for ERA-I (around 0.07). In ERA-I, the maximum is obtained over the Gulf of Mexico and north of $35^{\circ} \mathrm{N}$ toward the midlatitudes. The maximum TDV energy is obtained near Florida for TIE and is shifted northeastward in ENT and CAPENT, in better agreement with ERA-I. Near the African coast, the TDVs are moving northwestward in TIE and more westward in ERA-I 

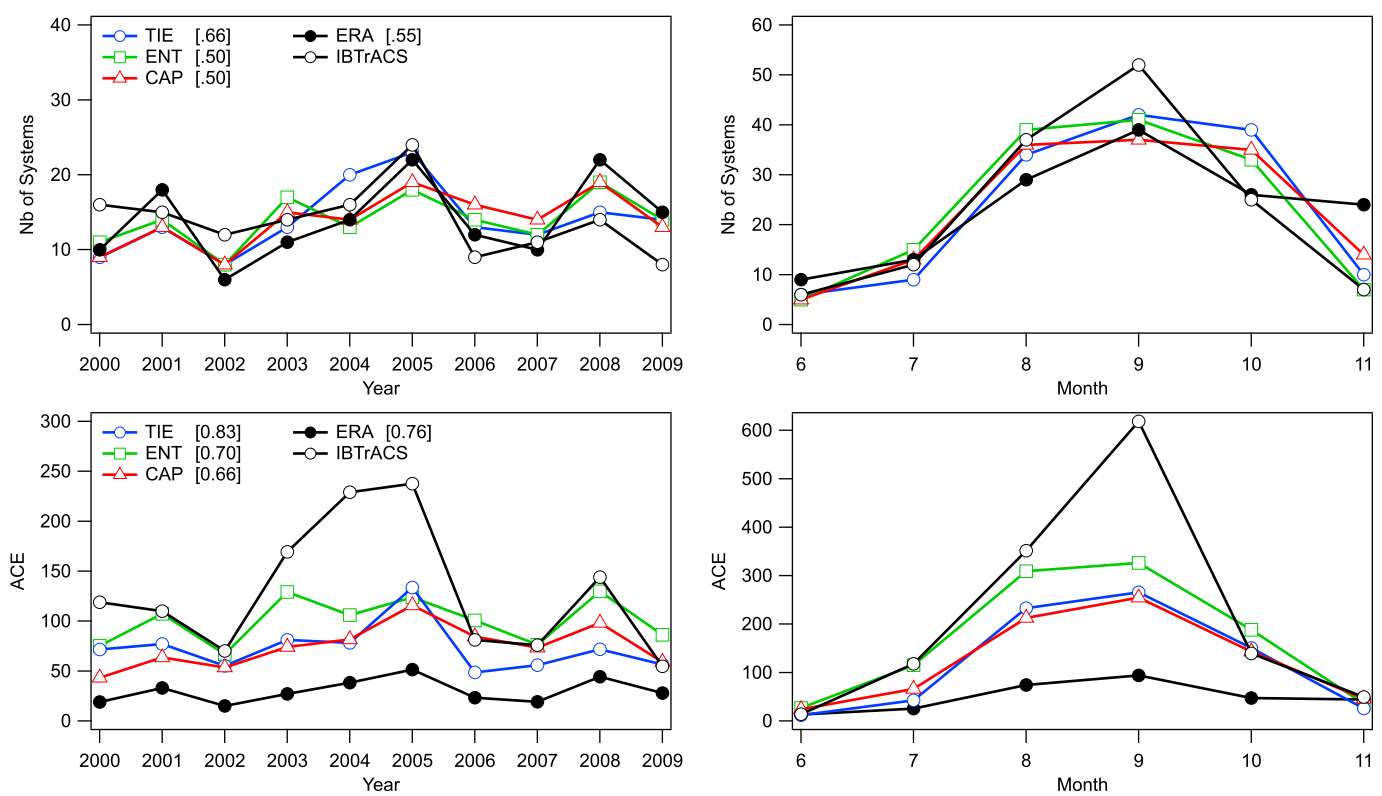

FIG. 5. Interannual and seasonal variations of the 140 strongest Atlantic TDVs and IBTrACS TCs for (top) the number of storms in IBTrACS, ERA-I, and LMDZ simulations; (bottom) the ACE of these systems. The numbers in brackets are the linear interannual correlation coefficients between ERA-I/LMDZ and IBTrACS for the number of TCs and ACE.

and CAPENT (even southwestward for some TDVs generated in the initiation corridor in ERA-I). In TIE, TDVs are thus driven away from regions with the highest SST, which may limit their growth and thus their number. The observed TC energy is an order of magnitude larger than the TDV energy in ERA-I and it maximizes over the Gulf of Mexico (around 0.6) and around $60^{\circ} \mathrm{W}$, south of the TDV energy maximum. The TC energy is stronger than the TDV energy in LMDZ, but the spatial distributions are very similar. This is expected, since the TCs detected by the CZ02 algorithm are indeed mostly the strongest TDVs, as shown in Fig. 2b. The TC energy is locally larger for TIE compared to ENT and CAPENT, confirming that the small TDV and TC numbers in TIE are mostly due to the initiation and intensification of early stage TDVs and not to the intensification of developed TDVs.

The seasonal distribution of occurrence and intensity of the $140 \mathrm{TDVs}$ with the largest ACE is quite well reproduced by LMDZ with the largest genesis occurrence in September (Fig. 5). The results are similar considering TCs detected with the CZ02 approach. Such a good agreement has been noted in previous studies, even in low-resolution models in some basins [see, e.g., Camargo and Sobel (2004) for the western North Pacific region]. In LMDZ, the number of TDV initiations is overestimated in October, but these TDVs have a relatively small ACE compared to August and September. The interannual correlation coefficients with observations are highest for
TIE (even larger than ERA-I). These coefficients are not significant at the 0.95 confidence level for the number of systems (except TIE), but they are significant for the ACE. The interannual distribution of the TDV occurrence in LMDZ shows some agreement with the observed IBTrACS and ERA-I distributions. LMDZ reproduces in particular the maxima in 2005 and 2008. Skill in reproducing aspects of Atlantic TC interannual variability in SST-forced AGCMs has already been noted in many studies (see, e.g., Roberts et al. 2015) and is certainly favored here by the wind nudging over West Africa, which gives more realistic eastern boundary conditions. However, the relatively large observed TC ACE in 2003 and 2004 are not depicted in either ERA-I or LMDZ. Some intensification processes, responsible for the strong TC ACE values in September and for years 2003-05, are clearly not represented in LMDZ and ERA-I. This suggests that TC intensification related to seasonal and interannual forcing is not taken into account in the simulations, possibly because of the absence of particular mesoscale processes.

\section{Potential physical sources of the differences between the simulations}

Analysis of the relative humidity ( $\mathrm{RH})$ profiles is used to diagnose how the different configurations of the convective scheme redistribute the water in the TDV column and in the environment. For three large regions 
(a) Western Atlantic

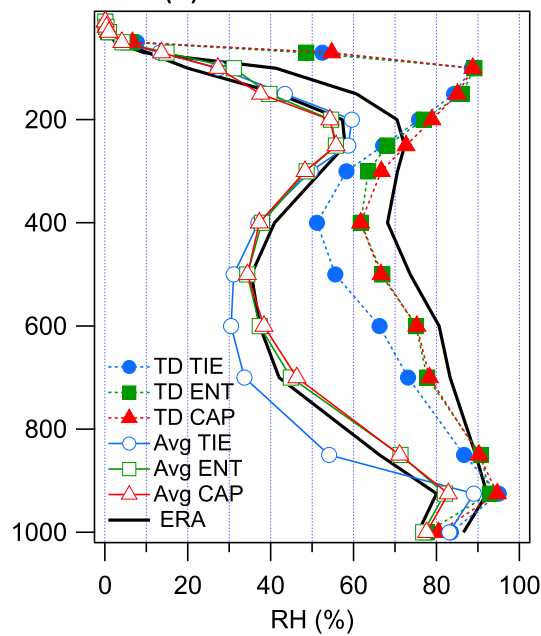

(b) Eastern Atlantic

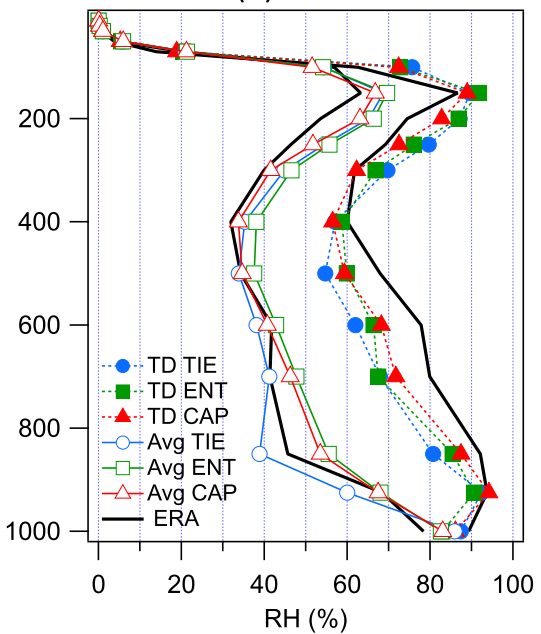

(c) Guinean region

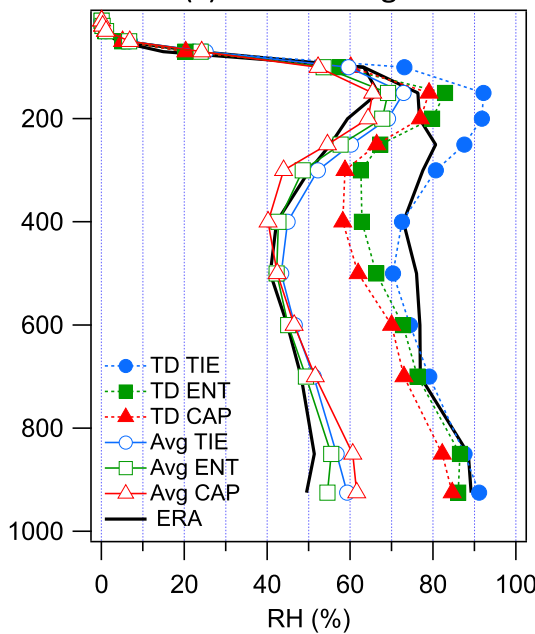

FIG. 6. Background and TDV RH profiles for the three regions defined in Fig. 3 and for the three simulations (TIE, ENT, and CAPENT) and ERA-I. Perturbed profiles (dotted lines and solid markers) are calculated by averaging the RH profiles over a square of $\pm 2.25^{\circ}$ ( \pm 3 grid points) around the TDV center for TDVs with $v_{\max }$ between 24 and $27 \mathrm{~m} \mathrm{~s}^{-1}$ for the western Atlantic; between 12 and $15 \mathrm{~m} \mathrm{~s}^{-1}$ for the eastern Atlantic; and between 3 and $6 \mathrm{~m} \mathrm{~s}^{-1}$ for the Guinean region. Background RH profiles are calculated by averaging monthly mean profiles for the same grid points (solid lines and open markers) for all TDVs.

(delineated by the boxes on the TIE field in Fig. 3), RH profiles in the TDVs are estimated by computing the average $\mathrm{RH}$ in squares of plus or minus three grid points around the TDV center. The associated background profile is computed by averaging the corresponding monthly mean profiles on the same grid points, giving a basic state weighted by the geographical and seasonal TDV occurrence. In Fig. 6, the TDV profiles are computed for TDVs with $v_{\max }$ between 24 and $27 \mathrm{~m} \mathrm{~s}^{-1}$ for the western Atlantic region; between 12 and $15 \mathrm{~m} \mathrm{~s}^{-1}$ for the eastern Atlantic region; and between 3 and $6 \mathrm{~m} \mathrm{~s}^{-1}$ for the Guinean region (the surface drag coefficient is larger for this continental region compared to ocean). These thresholds correspond to the largest $v_{\max }$ interval of $3 \mathrm{~m} \mathrm{~s}^{-1}$ with at least 10 TDVs for each simulation and for ERA-I.

For the western Atlantic, which is the region with the strongest simulated TDVs, with many strengthening into TCs (Fig. 4), the average moisture profiles for ENT and CAPENT are in good agreement with ERA-I, but the TIE profile is drier between 850 and $400 \mathrm{hPa}$ (Fig. 6a). This suggests that the smaller number of TDV genesis events over the western Atlantic Ocean for TIE could be related in part to the drier environment that inhibits convection, as discussed in Stan (2012), in a comparison to a model with a convective parameterization and one using "superparameterization." A limitation on such interpretations is that when the large-scale environment changes in response to a model physics change, it also results in changes to the dynamics of the disturbances (i.e., TDVs or TCs) and their sensitivities to the environment. One cannot necessarily assume that model-model environmental differences translate straightforwardly to differences in TDV or TC statistics, and environmental differences between low-resolution models has been previously found not to be predictive of differences in the statistics of TC-like disturbances (Camargo et al. 2007b). That said, convective inhibition associated with drier air in TIE may prevent the deepening of some early stage TDVs and thus limit their duration to less than 2 days, so they do not enter our sample. For developed TDVs $\left(24 \leq v_{\max } \leq\right.$ $27 \mathrm{~m} \mathrm{~s}^{-1}$ ), the TDV RH is too strong above $250 \mathrm{hPa}$ for the three LMDZ simulations compared to ERA-I. This shows that the altitude and the strength of the convective moisture detrainment in the central part of strong TDVs are overestimated by all three versions of the model (due to either resolved or parameterized convection). The TDV RH between 700 and $300 \mathrm{hPa}$ is larger in ERA-I, especially compared to TIE. The TDV moistening (i.e., the RH contrast between the background and the TDV) in the lower troposphere has a maximum near $600 \mathrm{hPa}$ for ENT, CAPENT, and ERA-I, and below $700 \mathrm{hPa}$ for TIE. The larger TDV moistening at low levels in TIE is consistent with the fact that some "premoistening" is probably necessary for the disturbance to become sufficiently moist to prevent the convective entrainment (related to the moisture divergence in TIE) from inhibiting the deep convection and the TDV growth.

Over the eastern Atlantic Ocean, developed TDVs come from the Guinean region in TIE (Figs. 3 and 4). Here, the average TIE moisture profile is closer to that of ERA-I with a relatively dry layer between 700 and $900 \mathrm{hPa}$ (Fig. 6b) that presumably inhibits 


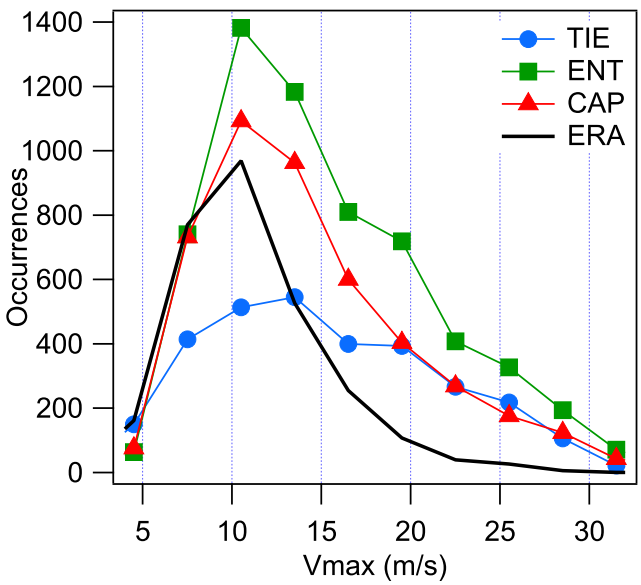

(a)
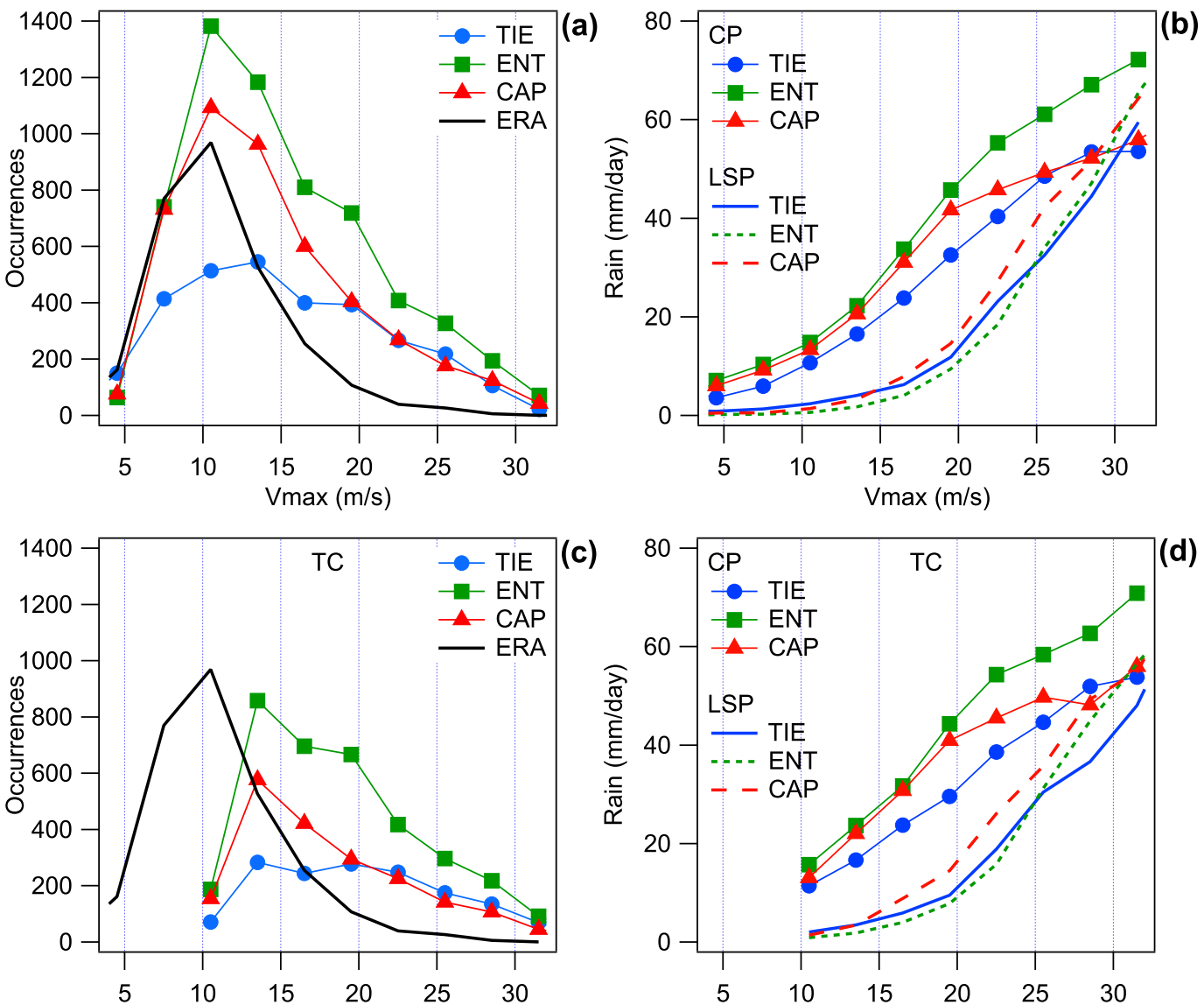

FIG. 7. (a) TDV occurrence as a function of $v_{\max }$ for the western Atlantic region for the three simulations. (b) As in (a), but for the rainfall rate from the convective parameterization (CP; line and marker) and from the large-scale condensation process (LSP; line). The rainfall rate is averaged in a square of $\pm 2.25^{\circ}$ ( \pm 3 grid points) around the TDV center. (c),(d) As in (a),(b), but for TC-like vortices.

convective development in this region. For developed TDVs $\left(12 \leq v_{\max } \leq 15 \mathrm{~m} \mathrm{~s}^{-1}\right)$, the moistening has a maximum near $850 \mathrm{hPa}$ for the three simulations and for ERA-I with the largest moistening occurring for ERA-I and TIE. The TDV RH is too large above $300 \mathrm{hPa}$ in all three simulations compared to ERA-I, suggesting again an excessive moisture detrainment at these levels in LMDZ at the expense of lower levels, and thus excessive deep convection at the expense of convection detraining at midlevel.

Over the Guinean region, TIE generates more Atlantic TDVs compared to ERA-I, ENT, and CAPENT. The lower troposphere is slightly moister on average in LMDZ compared to ERA-I but not significantly different in TIE. In the TDVs $\left(3 \leq v_{\max } \leq 6 \mathrm{~m} \mathrm{~s}^{-1}\right)$, the RH below $600 \mathrm{hPa}$ is also very similar among the three simulations and ERA-I (Fig. 6c), but the RH above $400 \mathrm{hPa}$ is larger for TIE and smaller for ENT and CAPENT compared to ERA-I. This suggests excessive deep convection in TIE and inhibited deep convection with the new entrainment that is larger in a dry environment. Because of the relatively large wind nudging in the eastern Guinean region, the TDV genesis is certainly similar for the three simulations there (this is a desired effect of the progressive wind nudging over West Africa). However, temperature and humidity are not substantially nudged, and differences in the convective scheme give indeed significant differences in the TDV evolution. The TDVs are sustained from their genesis over the Guinean region up to the Atlantic Ocean (where they spend at least 2 days based on the definition of the TDVs) for TIE, while they are more realistically inhibited with the new entrainment. This shows that, beyond the entrainment itself, the moisture content above West Africa may have a large influence on the origin of Atlantic TDVs.

One hypothesis evoked in the introduction is that the inhibition of the parameterized convection favors the triggering of resolved convection and gives higher TDV occurrence and intensity. This is analyzed in Fig. 7 by looking at the TDV/TC occurrence and TDV/TC 
rainfall as a function of $v_{\max }$ over the western Atlantic region. Compared to TIE, there are more TDV occurrences in ENT and CAPENT for $v_{\max }<20 \mathrm{~m} \mathrm{~s}^{-1}$ (Fig. 7a). For $v_{\max }>20 \mathrm{~m} \mathrm{~s}^{-1}$, the occurrences are, however, similar for TIE and CAPENT and slightly larger for ENT. This shows that the new entrainment increases mostly the number of TDV initiations over this region but not the intensification into stronger systems (forming inside or outside the region). For the three simulations, the TDV number ratio between LMDZ and ERA-I tends to increase exponentially with $v_{\max }$, confirming the overestimation of TDV intensification in LMDZ. Despite its lower TDV number, TIE is not in better agreement with ERA-I, since it gives too many strong TDVs $\left(v_{\max }>20 \mathrm{~m} \mathrm{~s}^{-1}\right)$ at the expense of weak and moderate TDVs.

The comparison between the rainfall rate due to the convective parameterization (CP) and the large-scale condensation process (LSP) gives information about the potential impact of the resolved convection on the TDV intensification (the hypothesis is that LSP rainfall results mostly from the resolved deep convective overturning in the heart of a TDV and not from low-level stratiform cloudiness). Figure $7 \mathrm{~b}$ shows that the LSP remains weak for the three LMDZ simulations for TDV with $v_{\max }<$ $20 \mathrm{~m} \mathrm{~s}^{-1}$. The LSP then increases more sharply and reaches a level comparable to that of the CP for $v_{\max }>$ $30 \mathrm{~m} \mathrm{~s}^{-1}$. The new entrainment formulation is not associated with larger LSP, showing that the difference in TDV numbers is not related to a higher occurrence of resolved convection. On the other hand, $\mathrm{CP}$ is larger for the new entrainment (ENT and CAPENT compared to TIE), especially for weak TDVs $\left(v_{\max }<20 \mathrm{~m} \mathrm{~s}^{-1}\right)$. This could explain why more TDVs can be sustained and reach the 2-day threshold with the new entrainment, with the larger CP favoring the deepening of weak TDVs. For CAPENT, CP tends to saturate for $v_{\max }>20 \mathrm{~m} \mathrm{~s}^{-1}$ and the rainfall rate intensification is mostly due to LSP. This suggests that the CAPE closure, in contrast to the lowlevel moisture convergence closure, gives more convective inhibition for intense TDVs and TC. For ENT, this saturation of the convective rain also occurs, but for larger values of $v_{\max }$. Therefore, the resolved convection is probably not the main reason for the larger TC and TDV numbers with the new entrainment. On the other hand, the new entrainment rate decreases when RH increases, leading to less diluted updrafts, larger $\mathrm{CP}$, and larger convective heating, which could deepen the TDVs. The moisture confinement effects of the TDVs lead indeed to large RH values close to the TDV center. In summary, the dry low-level troposphere in TIE may inhibit the convection and limit the deepening of early TDVs over the Atlantic Ocean. When the TDV is already developed, the large entrainment rate based on the moisture convergence above the cloud base limits the convective precipitation and thus the convective heating compared to the small entrainment rate based on $\mathrm{RH}$ (Fig. 7b). Selecting only TC-like vortices gives very similar results (Figs. 7c and 7d), showing that in LMDZ, most TDVs with $v_{\max }>10 \mathrm{~m} \mathrm{~s}^{-1}$ have a TC-like structure with a similar distribution of CP and LSP as a function of the vortex strength.

The difference between the TDV maintenance west of the Guinean region for the TIE simulation could be due to the representation of AEWs in the model. The AEWs in the simulations are analyzed using a local mode analysis (LMA; Goulet and Duvel 2000) with the multivariate approach (Duvel and Vialard 2007), which makes it possible to determine the perturbation pattern of a secondary parameter in regard to a reference parameter. The LMA analysis is done considering running time windows of 30 days and spectral harmonics 3-15 (i.e., periods of 2-10 days). The aim here is not to inspect in detail the LMA results but just to extract the average pattern of the AEWs by considering the meridional wind at $700 \mathrm{hPa}(\mathrm{V} 700)$ as the reference parameter and precipitation as the secondary parameter. Each average pattern shown in Fig. 8 is obtained from around $30 \mathrm{AEW}$ events with an average period around 4.5 days and a westward propagation speed around $9 \mathrm{~m} \mathrm{~s}^{-1}$, which corresponds well to observations, possibly thanks to the progressive wind nudging giving realistic boundary conditions to the east. Figure 8 shows average amplitude patterns for the three LMDZ simulations and for ERA-I (precipitations from GPCP). The wind pattern is quite realistic in the three simulations with a maximum near the African coast around $15^{\circ} \mathrm{N}$. The AEW signal is overestimated over the ocean for TIE and ENT in both V700 and precipitation, and is closer to ERA-I for CAPENT. In LMDZ, the AEW precipitation near the east African coast is overestimated compared to that in GPCP, in possible connection with the overestimated TDV initiation here (Fig. 3). The AEW amplitude for wind and precipitation are stronger west of the Guinean region for TIE compared to ENT and CAPENT. This suggests that the larger number of TDVs generated in the Guinean region and reaching the tropical Atlantic in TIE is related to the larger AEW signal near the coast, possibly because of a better phasing between the AEW dynamical and convective perturbations that deepen the TDVs associated with the AEWs. Further studies are required to determine how this could be related to the TIE entrainment. The continuity of the TDVs between the continent and the ocean is stronger for TIE than for ERAI probably because of the larger AEW amplitude near the coast. Note that for ERA-I, a larger number 

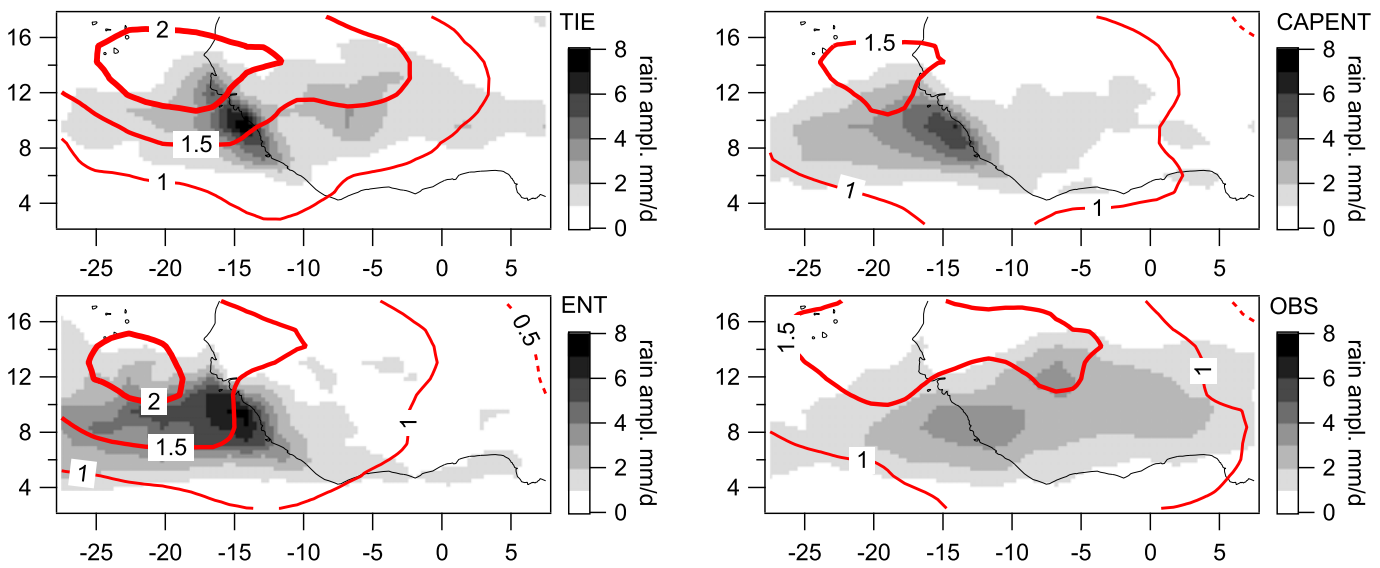

FIG. 8. Amplitude of the AEW signal for the three 10-yr LMDZ simulations between June and August for the meridional wind at $700 \mathrm{hPa}$ (contours) and rainfall rate (gray levels).

of TDVs are generated near the Hoggar Mountains, possibly related to AEWs, but this is beyond the scope of the present study.

\section{Summary and concluding remarks}

We used the zoom capability of LMDZ, with a typical resolution of $0.75^{\circ}$ over a large region of the North Atlantic and West Africa, to analyze the sensitivity of simulated tropical cyclone activity to entrainment and closure formulations used in the convection scheme. Two tracking approaches were used, one that detects tropical depression vortices (TDVs; Duvel 2015) and another that detects vortices exhibiting a more complete set of tropical cyclone (TC) characteristics, including a warm core (CZ02). The main advantage of the first approach is to provide continuous tracking of each TDV from a very early stage (over the ocean as well as over the African continent) up to its eventual extratropical transition, with very few a priori requirements on the TDV characteristics. From a methodological point of view, the TDV tracking technique is thus quite different from those used more traditionally for TC tracking and it is interesting to note the good agreement between the two approaches (Table 1). To a first approximation, the TCs detected by the CZ02 tracking scheme are also detected by the TDV tracking. A future possible development could be to join both approaches by adding structural criteria to detect TCs in the TDV tracks.

In most of the statistics evaluated from the distributions of simulated TDVs, the differences between LMDZ and ERA-I are larger than the differences between the three LMDZ simulations. The main difference among the three LMDZ simulations is the number of TDVs and TCs. In particular, the TIE simulation generates fewer TDVs and many fewer TCs compared to ENT and CAPENT. In the western Atlantic, the region of largest observed ACE, the TDV intensity distribution is, however, also clearly shifted toward intense TDVs in TIE (Fig. 7a). More generally, LMDZ overestimates the number of intense TDVs compared to ERA-I. In the western Atlantic, for example, the number of TDV occurrences is the same in ENT and ERA-I for $v_{\max }=10 \mathrm{~m} \mathrm{~s}^{-1}$, but there is a factor of 13 difference for $v_{\max }=25 \mathrm{~m} \mathrm{~s}^{-1}$. This exaggerated deepening of the TDVs in LMDZ is probably related in part to excessive deep convection at the expense of convection detraining at midlevels (Fig. 6). A striking result is that the weaker TDV intensity in ERA-I is associated with a larger propagation speed. Considering only the $\beta$ effect, weak TDVs in ERA-I should have smaller propagation speed compared to LMDZ and to observations. The large and realistic propagation speed in ERA-I could thus be due to either the more realistic steering flow or the assimilation procedure that tends to rectify the ERA-I TDV speed to the observed speed.

Our analysis suggests two factors that may explain the difference between the TDV intensity distributions in the different LMDZ simulations. First, the TIE simulation tends to dry the lower troposphere compared to the new entrainment. This dry lower troposphere, in better agreement with ERA-I over the eastern Atlantic, is likely to inhibit the convection in early stages of the TDV life cycle and thus decrease the number of TDVs reaching the 2-day duration threshold that are able to further intensify over the North Atlantic. Second, the parameterized convection in developed TDVs is stronger with the new entrainment for all TDV intensities, because it gives smaller entrainment rates for the high $\mathrm{RH}$ values that characterize the heart of the TDV. This parameterized convection "saturates" for relatively strong TDVs $\left(20 \mathrm{~m} \mathrm{~s}^{-1}\right.$ for CAPENT and around $25 \mathrm{~m} \mathrm{~s}^{-1}$ for ENT) 
but is still more active than the resolved convection. The higher TDV intensity with the new entrainment is thus not due to the inhibition of the parameterized convection.

The TDV tracking also gives an estimate of the different potential sources of TCs over the North Atlantic and the sensitivity of these sources to the convective parameterization. The TDV sources are very different between TIE and the two other simulations despite the wind nudging over West Africa. The genesis of TDVs over the Atlantic is very rare in TIE, while the number of TDVs generated over West Africa and reaching the tropical Atlantic is much larger in that simulation than in the others (or in ERA-I); interestingly, the 20 strongest TDVs are all generated over West Africa in TIE. This appears to be related to a larger AEW activity in TIE, which could be related to a better phasing between the convective activity and the wave trough over continental regions.

Our analysis shows the complexity involved in diagnosing the differences in the TDV distribution among different versions of a model. Differences arise not only from the modification of the physical processes in the TDVs and TCs themselves but also from differences in the average thermodynamic and dynamic structures of the atmosphere. These differences may play a role in the TDV and TC genesis location and frequency, as well as in the intensification and maintenance processes. This demonstrates that the separation between TC initiation and intensification processes may help to understand the sources of TC activity biases in a model and to interpret the differences among models.

Acknowledgments. The authors thank the LMD modeling group and especially F. Hourdin and L. Li for advice about the zoomed version of the LMDZ, and L. Guez for having verified the code for the new versions of the convection scheme and carried out the simulations. This research was partially supported by a grant from the Alliance Program at Columbia University, which enabled JPD to visit Columbia and SJC to visit LMD. SJC and AHS acknowledge support from NSF Grant AGS 1143959 and NOAA Grant NA110AR4310093.

\section{REFERENCES}

Bechtold, P., M. Köhler, T. Jung, F. Doblas-Reyes, M. Leutbecher, M. Rodwell, F. Vitart, and G. Balsamo, 2008: Advances in simulating atmospheric variability with the ECMWF model: From synoptic to decadal time-scales. Quart. J. Roy. Meteor. Soc., 134, 1337-1351, doi:10.1002/qj.289.

, N. Semane, P. Lopez, J. P. Chaboureau, A. Beljaars, and N. Bormann, 2014: Representing equilibrium and nonequilibrium convection in large-scale models. J. Atmos. Sci., 71, 734-753, doi:10.1175/JAS-D-13-0163.1.
Bell, G. D., and Coauthors, 2000: Climate assessment for 1999. Bull. Amer. Meteor. Soc., 81, S1-S50, doi:10.1175/ 1520-0477(2000)81[s1:CAF]2.0.CO;2.

Burpee, R. W., 1972: The origin and structure of easterly waves in the lower troposphere of North Africa. J. Atmos. Sci., 29, 77-90, doi:10.1175/1520-0469(1972)029<0077:TOASOE > 2.0.CO;2.

__, 1975: Some features of synoptic-scale waves based on a compositing analysis of GATE data. Mon. Wea. Rev., 103, 921-925, doi:10.1175/1520-0493(1975)103<0921: SFOSWB $>2.0 . \mathrm{CO} ; 2$.

Camargo, S. J., 2013: Global and regional aspects of tropical cyclone activity in the CMIP5 models. J. Climate, 26, 9880-9902, doi:10.1175/JCLI-D-12-00549.1.

— tracking of tropical cyclones in atmospheric general circulation models. Wea. Forecasting, 17, 1152-1162, doi:10.1175/ 1520-0434(2002)017<1152:ITDATO > 2.0.CO;2.

—, and A. H. Sobel, 2004: Formation of tropical storms in an atmospheric general circulation model. Tellus, 56A, 56-67, doi:10.1111/j.1600-0870.2004.00033.x.

—_, and A. G. Barnston, 2009: Experimental seasonal dynamical forecasts of tropical cyclone activity at IRI. Wea. Forecasting, 24, 472-491, doi:10.1175/2008WAF2007099.1.

_ Wiley Interdiscip. Rev.: Climate Change, 7, 211-237, doi:10.1002/wcc. 373 .

— A. A. Barnston, and S. E. Zebiak, 2005: A statistical assessment of tropical cyclone activity in atmospheric general circulation models. Tellus, 57A, 589-604, doi:10.1111/ j.1600-0870.2005.00117.x.

_ H. Li, and L. Sun, 2007a: Feasibility study for downscaling seasonal tropical cyclone activity using the NCEP regional spectral model. Int. J. Climatol., 27, 311-325, doi:10.1002/ joc. 1400 .

_ A. H. Sobel, A. Barnston, and K. A. Emanuel, 2007b: Tropical cyclone genesis potential in climate models. Tellus, 59A, 428-443, doi:10.1111/j.1600-0870.2007.00238.x.

Carlson, T. N., 1969: Synoptic histories of three African disturbances that developed into Atlantic hurricanes. Mon. Wea. Rev., 97, 256-276, doi:10.1175/1520-0493(1969)097<0256: SHOTAD $>2.3 . \mathrm{CO} ; 2$.

Caron, L. P., and C. Jones, 2012: Understanding and simulating the link between African easterly waves and Atlantic tropical cyclones using a regional climate model: The role of domain size and lateral boundary conditions. Climate Dyn., 39, 113-135, doi:10.1007/s00382-011-1160-8.

Daloz, A. S., F. Chauvin, K. Walsh, S. Lavender, D. Abbs, and F. Roux, 2012: The ability of general circulation models to simulate tropical cyclones and their precursors over the North Atlantic main development region. Climate Dyn., 39, 15591576, doi:10.1007/s00382-012-1290-7.

Davis, C., C. Snyder, and A. C. Didlake Jr., 2008: A vortex-based perspective of eastern Pacific tropical cyclone formation. Mon. Wea. Rev., 136, 2461-2477, doi:10.1175/2007MWR2317.1.

Dee, D. P., and Coauthors, 2011: The ERA-Interim reanalysis: Configuration and performance of the data assimilation system. Quart. J. Roy. Meteor. Soc., 137, 553-597, doi:10.1002/qj.828.

Dunkerton, T. J., M. T. Montgomery, and Z. Wang, 2009: Tropical cyclogenesis in a tropical wave critical layer: Easterly waves. Atmos. Chem. Phys., 9, 5587-5646, doi:10.5194/ acp-9-5587-2009.

Duvel, J.-P., 2015: Initiation and intensification of tropical depressions over the southern Indian Ocean: Influence of the 
MJO. Mon. Wea. Rev., 143, 2170-2191, doi:10.1175/ MWR-D-14-00318.1.

_ , and J. Vialard, 2007: Indo-Pacific sea surface temperature perturbations associated with intraseasonal oscillations of the tropical convection. J. Climate, 20, 3056-3082, doi:10.1175/ JCLI4144.1.

Emanuel, K. A., 1991: A scheme for representing cumulus convection in large-scale models. J. Atmos. Sci., 48, 2313-2329, doi:10.1175/1520-0469(1991)048<2313:ASFRCC >2.0.CO;2.

Goulet, L., and J.-P. Duvel, 2000: A new approach to detect and characterize intermittent atmospheric oscillations: Application on the intraseasonal oscillation. J. Atmos. Sci., 57, 2397-2416, doi:10.1175/1520-0469(2000)057<2397: ANATDA $>2.0 . \mathrm{CO} ; 2$.

He, F., and D. J. Posselt, 2015: Impact of parameterized physical processes on simulated tropical cyclone characteristics in the Community Atmosphere Model. J. Climate, 28, 9857-9872, doi:10.1175/JCLI-D-15-0255.1.

Hourdin, F., and Coauthors, 2006: The $\mathrm{LMDZ}_{4}$ general circulation model: Climate performance and sensitivity to parametrized physics with emphasis on tropical convection. Climate Dyn., 27, 787-813, doi:10.1007/s00382-006-0158-0.

Kim, D., and Coauthors, 2012: The tropical subseasonal variability simulated in the NASA GISS general circulation model J. Climate, 25, 4641-4659, doi:10.1175/JCLI-D-11-00447.1.

Knapp, K. R., M. C. Kruk, D. H. Levinson, H. J. Diamond, and C. J. Neumann, 2010: The International Best Track Archive for Climate Stewardship (IBTrACS): Unifying tropical cyclone data. Bull. Amer. Meteor. Soc., 91, 363-376, doi:10.1175/ 2009BAMS2755.1.

Landman, W. A., A. Seth, and S. J. Camargo, 2005: The effect of regional climate model domain choice on the simulation of tropical cyclone-like vortices in the southwestern Indian Ocean. J. Climate, 18, 1263-1274, doi:10.1175/JCLI3324.1.

Landsea, C. W., 1993: A climatology of intense (or major) Atlantic hurricanes. Mon. Wea. Rev., 121, 1703-1713, doi:10.1175/ 1520-0493(1993)121<1703:ACOIMA > 2.0.CO;2.

Liebmann, B., H. H. Hendon, and J. D. Glick, 1994: The relationship between tropical cyclones of the western Pacific and Indian Oceans and the Madden-Julian oscillation. J. Meteor. Soc. Japan, 72, 401-411.

Martin, E. R., and C. Thorncroft, 2015: Representation of African easterly waves in CMIP5 models. J. Climate, 28, 7702-7715, doi:10.1175/JCLI-D-15-0145.1.

Maue, R. N., 2009: Northern Hemisphere tropical cyclone activity. Geophys. Res. Lett., 36, L05805, doi:10.1029/2008GL035946.

Mozer, J. B., and J. A. Zehnder, 1996: Lee vorticity production by large-scale tropical mountain ranges. Part I: Eastern North
Pacific tropical cyclogenesis. J. Atmos. Sci., 53, 521-538, doi:10.1175/1520-0469(1996)053<0521:LVPBLS>2.0.CO;2.

Murakami, H., and Coauthors, 2012a: Future changes in tropical cyclone activity projected by the new high-resolution MRI-AGCM. J. Climate, 25, 3237-3260, doi:10.1175/ JCLI-D-11-00415.1.

— , R. Mizuta, and E. Shindo, 2012b: Future changes in tropical cyclone activity project by multi-physics and multi-SST ensemble experiments using 60-km-mesh MRI-AGCM. Climate Dyn., 39, 2569-2584, doi:10.1007/s00382-011-1223-x.

Reed, K. A., and C. Jablownowski, 2011: Impact of physical parametrization on idealized tropical cyclones in the Community Atmosphere Model. Geophys. Res. Lett., 38, L048045, doi:10.1029/2010GL046297.

Reed, R., D. Norquist, and E. Recker, 1977: The structure and properties of African wave disturbances as observed during Phase III of GATE12. Mon. Wea. Rev., 105, 317-333, doi:10.1175/1520-0493(1977)105<0317:TSAPOA >2.0.CO;2.

Roberts, M., and Coauthors, 2015: Tropical cyclones in the UPSCALE ensemble of high-resolution global climate models. J. Climate, 28, 574-59, doi:10.1175/JCLI-D-14-00131.1.

Shaevitz, D. A., and Coauthors, 2014: Characteristics of tropical cyclones in high-resolution models of the present climate. J. Adv. Model. Earth Syst., 6, 1154-1172, doi:10.1002/2014MS000372.

Smith, R. B., 1993: A hurricane beta-drift law. J. Atmos. Sci., 50, 3213-3215, doi:10.1175/1520-0469(1993)050<3213: AHBDL $>2.0 . \mathrm{CO} ; 2$.

Stan, C., 2012: Is cumulus convection the concertmaster of tropical cyclone activity in the Atlantic? Geophys. Res. Lett., 39, L19716, doi:10.1029/2012GL053449.

Tiedtke, M., 1989: A comprehensive mass flux scheme for cumulus parameterization in large-scale models. Mon. Wea. Rev., 117, 1779-1800, doi:10.1175/1520-0493(1989)117<1779: ACMFSF $>2.0 . \mathrm{CO} ; 2$

Vitart, F., J. L. Anderson, J. Sirutis, and R. E. Tuleya, 2001: Sensitivity of tropical storms simulated by a general circulation model to changes in cumulus parametrization. Quart. J. Roy. Meteor. Soc., 127, 25-51, doi:10.1002/qj.49712757103.

Walsh, K. J. E., and Coauthors, 2016: Tropical cyclones and climate change. Wiley Interdiscip. Rev.: Climate Change, 7, 65-89, doi:10.1002/wcc.371.

Yang, H., Z. Jiang, and L. Li, 2016: Biases and improvements in three dynamical downscaling climate simulations over China. Climate Dyn., 47, 3235-3251, doi:10.1007/s00382-016-3023-9.

Zhao, M., I. M. Held, and S.-J. Lin, 2012: Some counterintuitive dependencies of tropical cyclone frequency on parameters in a GCM. J. Atmos. Sci., 69, 2272-2283, doi:10.1175/ JAS-D-11-0238.1. 